\title{
Enhancing Corrosion Inhibition Performance of ZnO Solid Solution by Doping Variable-Valence Rare-Earth Element Cerium
}

\author{
Si-Rui Zhao ${ }^{1}$, Xiao-Yu Yuan ${ }^{1}$, Yi-Xiang Chen ${ }^{1}$, Yi Lu ${ }^{1,2}$, Min Zhang ${ }^{1}$, Jin-Ku Liu ${ }^{1,2, *}$ \\ ${ }^{1}$ School of Chemistry and Molecular Engineering, East China University of Science and
}

Technology, Shanghai, 200237, P.R. China

${ }^{2}$ Material Corrosion and Protection Key Laboratory of Sichuan Province, Sichuan University of

Science and Engineering, Zigong, Sichuan 643000, P. R. China

\footnotetext{
* Corresponding author; E-mail address: jkliu@ecust.edu.cn (Jin-Ku Liu)
} 


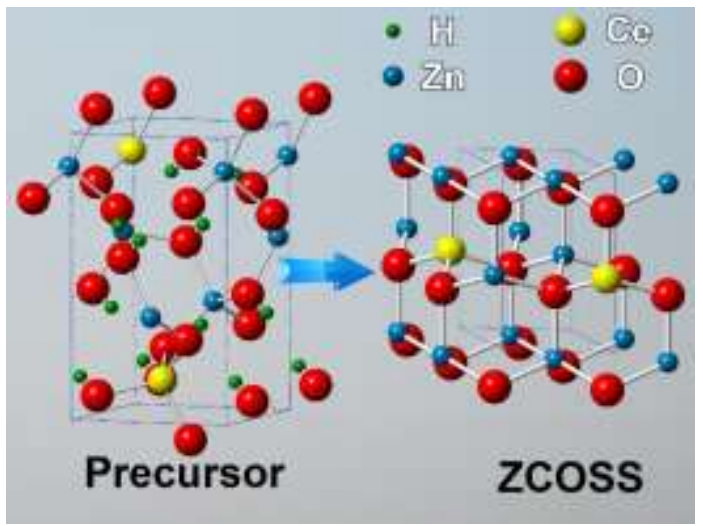

S1. Diagrammatic crystal structure of the precursor and the ZCOSS material.

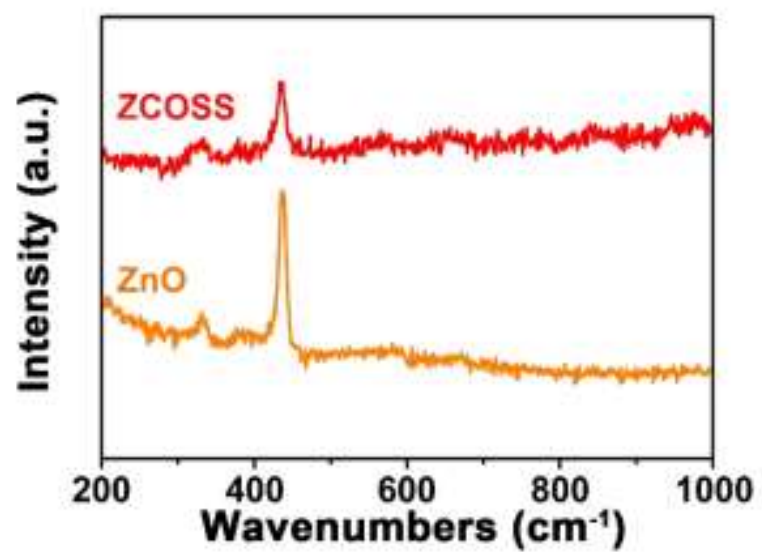

S2. The room-temperature Raman spectra of representative $\mathrm{ZnO}$ and the ZCOSS materials. As can be seen from the obtained spectrum the intense and sharp narrow peak at 437.6 $\mathrm{cm}^{-1}$ corresponds to a nonpolar optical phonon $\mathrm{E}_{2}$ (high) of wurtzite $\mathrm{ZnO}$ while the 331.9 $\mathrm{cm}^{-1}$ is assigned to the second-order Raman spectrum arising from zone boundary phonons of the hexagonal $\mathrm{ZnO}$. As compared with the $\mathrm{ZnO}$ material, the $\mathrm{E}_{2}$ (high) modes of the ZCOSS material shifted from 437.6 to $435.0 \mathrm{~cm}^{-1}$, and its intensity decreased. The shift is believed to originate from the residual stress along the $\mathrm{c}$ axis due to the lattice distortion. 
S3. Electrochemical parameters extracted from impedance data for the steel plate coated with epoxy resin coating immersion in $3.5 \mathrm{wt} \% \mathrm{NaCl}$ solution

\begin{tabular}{ccccccccc} 
Sample & $\begin{array}{c}\mathrm{R}_{\mathrm{s}} / \\
\mathrm{ohm} \cdot \mathrm{cm}^{-2}\end{array}$ & $\begin{array}{c}\mathrm{Y}_{1} / \mathrm{ohm} \\
1 \cdot \mathrm{cm}^{-2} \cdot \mathrm{s}^{-\mathrm{n}}\end{array}$ & $\mathrm{n}_{1}$ & $\begin{array}{c}\mathrm{R}_{\mathrm{ct}} / \\
\mathrm{ohm} \cdot \mathrm{cm}^{-2}\end{array}$ & $\begin{array}{c}\mathrm{Y}_{1} / \mathrm{ohm}^{-} \\
1 \cdot \mathrm{cm}^{-2} \cdot \mathrm{s}^{-\mathrm{n}}\end{array}$ & $\mathrm{n}_{2}$ & $\begin{array}{c}\mathrm{R}_{\mathrm{f}} / \\
\mathrm{ohm} \cdot \mathrm{cm}^{-2}\end{array}$ & $\begin{array}{c}\mathrm{Z} / \\
\mathrm{ohm} \cdot \mathrm{cm}^{-2}\end{array}$ \\
\hline Epoxy resin (6 h) & 10.46 & $1.841 \times 10^{-4}$ & 0.6120 & 7194 & $1.478 \times 10^{-4}$ & 0.5479 & 35.90 & 4627.1 \\
Epoxy resin (24 h) & 10.37 & $4.201 \times 10^{-4}$ & 0.6508 & 2609 & $6.126 \times 10^{-4}$ & 0.4758 & 25.71 & 1870.1 \\
Epoxy resin (48 h) & 20.30 & $3.245 \times 10^{-4}$ & 0.7715 & 1665 & $7.347 \times 10^{-4}$ & 0.4657 & 41.25 & 1530.9 \\
Epoxy resin (72 h) & 10.43 & $9.220 \times 10^{-4}$ & 0.7007 & 1552 & $1.333 \times 10^{-3}$ & 0.4538 & 28.03 & 1095.0 \\
\hline
\end{tabular}

S4. Electrochemical parameters extracted from impedance data for the steel plates coated with $\mathrm{ZnO}$ and the $\mathrm{ZCOSS}$ composite system immersion in $3.5 \mathrm{wt} \% \mathrm{NaCl}$ solutions

\begin{tabular}{|c|c|c|c|c|c|c|c|c|c|c|}
\hline Sample & $\begin{array}{c}\mathrm{R}_{\mathrm{s}} / \\
\mathrm{ohm} \cdot \mathrm{cm}^{-2}\end{array}$ & $\begin{array}{l}\mathrm{Y}_{1} / \mathrm{ohm}^{-} \\
{ }^{1} \cdot \mathrm{cm}^{-2} \cdot \mathrm{s}^{-\mathrm{n}}\end{array}$ & $\mathrm{n}_{1}$ & $\begin{array}{c}\mathrm{R}_{\mathrm{c}} / \\
\mathrm{ohm} \cdot \mathrm{cm}^{-2}\end{array}$ & $\begin{array}{l}\mathrm{Y}_{2} / \mathrm{ohm}^{-} \\
{ }^{1} \cdot \mathrm{cm}^{-2} \cdot \mathrm{s}^{-\mathrm{n}}\end{array}$ & $\mathrm{n}_{2}$ & $\begin{array}{c}\mathrm{R}_{\mathrm{ct}} / \\
\mathrm{ohm} \cdot \mathrm{cm}^{-2}\end{array}$ & $\mathrm{C}_{\mathrm{f}} / \mathrm{F} \cdot \mathrm{cm}^{-2}$ & $\begin{array}{c}\mathrm{R}_{\mathrm{f}} / \\
\mathrm{ohm} \cdot \mathrm{cm}^{-2}\end{array}$ & $\begin{array}{c}\mathrm{Z} / \\
\mathrm{ohm} \cdot \mathrm{cm}^{-2}\end{array}$ \\
\hline $\mathrm{ZnO}(6 \mathrm{~h})$ & 20.41 & $1.326 \times 10^{-6}$ & 0.8565 & 189.2 & $3.443 \times 10^{-5}$ & 0.5688 & 6117 & $2.111 \times 10^{-4}$ & 5136 & 10900 \\
\hline ZCOSS (6 h) & 16.21 & $1.727 \times 10^{-5}$ & 0.7719 & $1219 \times 10^{1}$ & $6.540 \times 10^{-5}$ & 0.4899 & $3559 \times 10^{1}$ & $8.994 \times 10^{-6}$ & 1027 & 27901 \\
\hline $\mathrm{ZnO}(24 \mathrm{~h})$ & 18.96 & $4.849 \times 10^{-6}$ & 0.7956 & 145.2 & $5.828 \times 10^{-5}$ & 0.6808 & 4374 & $5.799 \times 10^{-4}$ & 2611 & 6790.6 \\
\hline $\mathrm{ZCOSS}(24 \mathrm{~h})$ & 18.54 & $6.505 \times 10^{-5}$ & 0.5534 & 115.1 & $3.004 \times 10^{-5}$ & 0.7512 & $1498 \times 10^{1}$ & $3.484 \times 10^{-5}$ & 606.9 & 14104 \\
\hline $\mathrm{ZnO}(48 \mathrm{~h})$ & 21.98 & $1.831 \times 10^{-5}$ & 0.7003 & 88.42 & $1.539 \times 10^{-4}$ & 0.6429 & 3469 & $1.300 \times 10^{-4}$ & 436.9 & 3412.6 \\
\hline $\mathrm{ZCOSS}(48 \mathrm{~h})$ & 21.84 & $1.756 \times 10^{-4}$ & 0.4772 & 132.8 & $4.603 \times 10^{-5}$ & 0.7574 & 9717 & $9.571 \times 10^{-6}$ & 38.50 & 8890.5 \\
\hline $\mathrm{ZnO}(72 \mathrm{~h})$ & 25.78 & $1.116 \times 10^{-4}$ & 0.5397 & 38.76 & $1.871 \times 10^{-4}$ & 0.6716 & 4485 & $4.637 \times 10^{-4}$ & 237.9 & 3744.5 \\
\hline $\operatorname{ZCOSS}(72 h)$ & 31.95 & $4.282 \times 10^{-5}$ & 0.6500 & 151.8 & $6.516 \times 10^{-5}$ & 0.8064 & 7204 & $7.019 \times 10^{-7}$ & 5.617 & 6758.1 \\
\hline
\end{tabular}

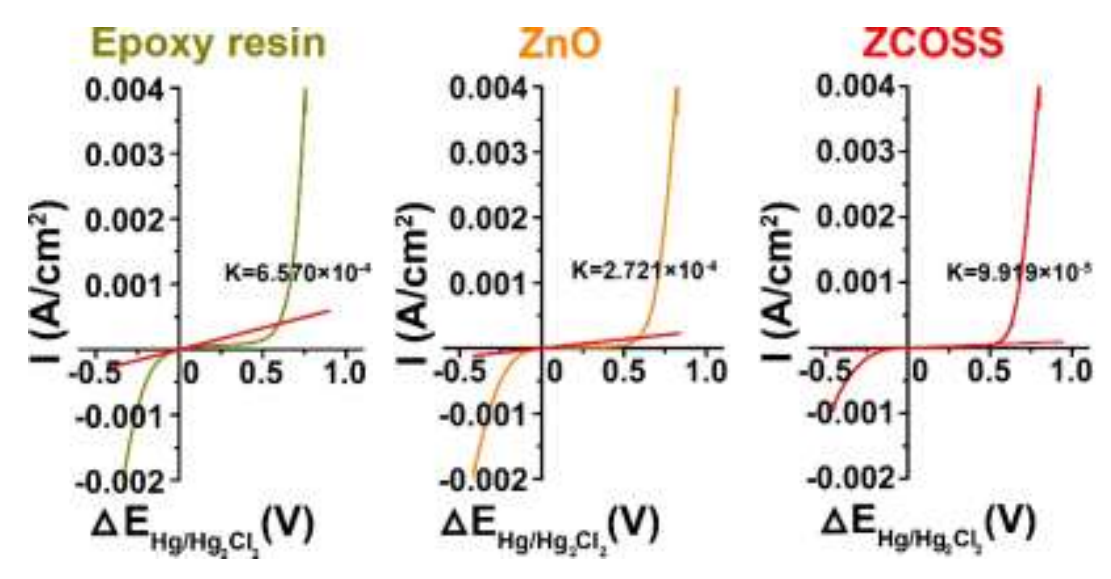


S5. Tafel polarization curves $(\Delta \mathrm{E}-\mathrm{I})$ of the shielded layers after immersion in $3.5 \mathrm{wt} \%$

$\mathrm{NaCl}$ solutions for $72 \mathrm{~h}$.
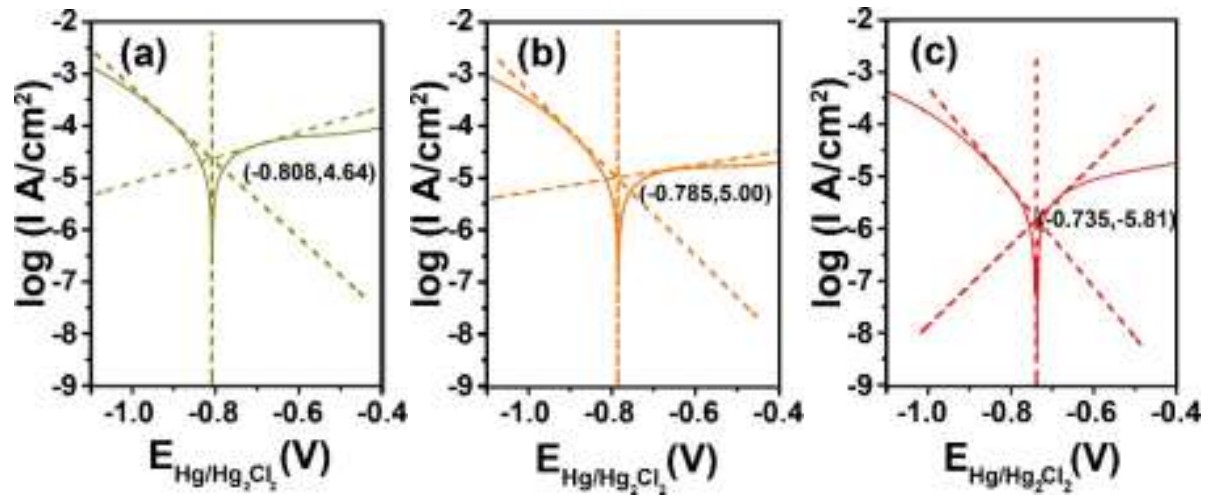

S6. Tafel polarization curves (E-log I) of (a) epoxy resin coating; (b) $\mathrm{ZnO}$, and (c) the ZCOSS shielded layers.

S7. Experimental details of the BET measurement

The BET tests were based on the Micromeritics ASAP 2400 instrument and its workstation.

Pretreatment part. 1 . atmospheric heating: treatment temperature: $100^{\circ} \mathrm{C}$, treatment time: $60 \mathrm{~min}$.

2. Vacuum heating: processing temperature; $200^{\circ} \mathrm{C}$, processing time: $200 \mathrm{~min}$, number of pumping: 100 times, number of inflation: 80 times, inflation temperature: $40{ }^{\circ} \mathrm{C}$, temperature rise rate: $1^{\circ} \mathrm{C} / \mathrm{min}$.

Test part.1. Gas mode: nitrogen.

2. sample parameters: sample tube volume: $35.000 \mathrm{ml}$, sample mass: $2.00000 \mathrm{~g}$.

3. correction parameters: volume correction parameter: 0.075 .

4. Test parameters: $\mathrm{P} / \mathrm{P}_{0}$ selection point: automatic selection point; $\mathrm{P}_{0}$ value determination: fixed pre-value; inflation mode: intelligent calculation; liquid nitrogen surface control: enabled; automatic backfill inflation: enabled.

Calculation part. The points between $\mathrm{P} / \mathrm{P}_{0}=0.05-0.35$ are selected to calculate the specific surface area of the material by the following formula. 


$$
\frac{P}{V_{d}\left(P_{0}-P\right)}=\frac{1}{V_{m} C}+\frac{C-1}{V_{m} C} \frac{P}{P_{0}}
$$

where $V_{d}$ is the adsorption volume of $\mathrm{N}_{2}$ at a relative pressure of $P_{0} ; V_{m}$ is the average adsorption volume of adsorbed molecules in a single molecular layer on the surface of the test material; $P_{0}$ is the vapor pressure of the adsorbent at the adsorption temperature when in the condensed state; $C$ is a physical constant related to the heat of adsorption $(\Delta \mathrm{H})$ and the heat of condensation of the adsorbent $\left(\Delta \mathrm{H}_{\mathrm{c}}\right)$.

S8. Calcination conditions

\begin{tabular}{lccc} 
Substance & $\begin{array}{c}\text { Calcination temperature } \\
/{ }^{\circ} \mathrm{C}\end{array}$ & $\begin{array}{c}\text { Nitrogen } \\
\text { protection }\end{array}$ & $\begin{array}{c}\text { Calcination time / } \\
\text { min }\end{array}$ \\
ZCOSS1 & 300 & & \\
ZCOSS2 & 400 & Yes & \\
ZCOSS3 & 500 & & $30 \mathrm{~min}$ \\
\cline { 2 - 3 } ZCOSS4 & 300 & & \\
ZCOSS5 & 400 & No & \\
ZCOSS6 & 500 & & \\
\hline
\end{tabular}

